\title{
Unveiling square and triangular optical lattices: a comparative study
}

\author{
Juarez G. Silva, ${ }^{1}$ Alcenísio J. Jesus-Silva, ${ }^{1}$ Márcio A. R. C. Alencar, ${ }^{2,1}$ \\ Jandir M. Hickmann, ${ }^{3,1}$ and Eduardo J. S. Fonseca ${ }^{1, *}$ \\ ${ }^{1}$ Optics and Materials Group-OPTMA, Universidade Federal de Alagoas, Caixa Postal 2051, Maceió, AL 57061-970, Brazil \\ ${ }^{2}$ Departamento de Fisica, Universidade Federal de Sergipe, Centro de Ciências Exatas e Tecnologia, \\ Cidade Universitária Prof. José Aloísio de Campos, Rod. Marechal Rondon s/n, Jardim Rosa Elze, \\ São Cristóvão, SE 57072-970, Brazil \\ ${ }^{3}$ Instituto de Física, Universidade Federal do Rio Grande do Sul, 91501-970, Porto Alegre, RS, Brazil \\ *Corresponding author: efonseca@optma.org
}

Received October 28, 2013; revised January 7, 2014; accepted January 7, 2014; posted January 8, 2014 (Doc. ID 200301); published February 11, 2014

\begin{abstract}
We study square and triangular optical lattice formation using a diffraction technique with light-possessing orbital angular momentum (OAM). We demonstrate that it is possible to use Fraunhofer diffraction of light by a square aperture to unveil OAM about two times bigger than would be possible with a triangular aperture. We notice that the pattern remains truncated until a topological charge (TC) equal to 20 with good precision. Even though a square pattern cannot be used to determine the TC sign, it is possible to measure high order of the modulus and sign of the TC up to 20, combining patterns of the triangular and square apertures. (c) 2014 Optical Society of America

OCIS codes: (050.0050) Diffraction and gratings; (050.4865) Optical vortices; (050.1940) Diffraction; (050.1220) Apertures.

http://dx.doi.org/10.1364/OL.39.000949
\end{abstract}

Light beams with orbital angular momentum (OAM) are associated with an azimuthal phase structure $\exp (i m \phi)$, where $m$ is called topological charge (TC). This phase is responsible for rotating the Poynting vector. High-order Laguerre-Gauss (LG) [1] and high-order Bessel [2] beams are examples of beams carrying OAM.

Since the seminal work by Allen et al. [3] ], OAM have been extensively explored in light-matter interaction [ $\underline{4}-7]$ and diffraction phenomena [ $8-10]$. Other applications of light's OAM range from optical manipulation [11] to quantum communication $[12,13]$. Two recent publications show the importance of this subject applied to optical communications [14] and quantum metrology [15].

Particularly interesting is the rich relationship between the phase of light with OAM and diffraction phenomena $[9,10,16-20]$. This relationship was well explored by a very simple experiment performed by Hickmann et al. [10]. The basic idea is to observe the Fraunhofer pattern of a diffracted light with OAM by a triangular slit or triangular aperture with the phase singularity aligned on the center of these objects. A truncated triangular optical lattice in the Fraunhofer plane is observed. The size of this optical lattice depends on the amount of OAM, and by counting the number of intensity maxima $N$ of any extern side of the triangular lattice you can obtain the value of TC, $m$, using a very simple rule, $m=N-1$. A simple way to understand the formation of this pattern is to observe the diffraction of light with OAM due to each edge of the aperture separately in Fraunhofer plane. Two points must be observed: firstly, the number of fringes due to each edge is proportional to the OAM value, and second, the effect of the azimuthal phase over this diffraction pattern produces a shift proportional to the amount of OAM. By interfering the light diffracted by the three edges, a triangular optical lattice is unveiled. In fact, in [9] the authors showed a detailed study of the diffraction problem of light with OAM by a single slit.
They considered two situations where the phase singularity of the light beam strikes on the center of and above a single slit. In the latter case, which is the case for one side of the triangular aperture, the patterns observed are asymmetric and shifted.

At this point a very simple question arises: What can we learn about diffracting OAM beam by other polygonal shapes? In [20], results of diffraction of light with OAM by a square aperture were presented. The authors showed numerically and experimentally that a perfect square optical intensity lattice takes place only for even values of the TC.

In this Letter, we show a comparative study of the diffraction problem of light with OAM between square and triangular shape. Surprisingly, with a square aperture the value of TC obtained outperforms for more than two times the maximum value of TC using a triangular aperture. We present experimental results, computer simulations, and a heuristic argument that explains this observation.

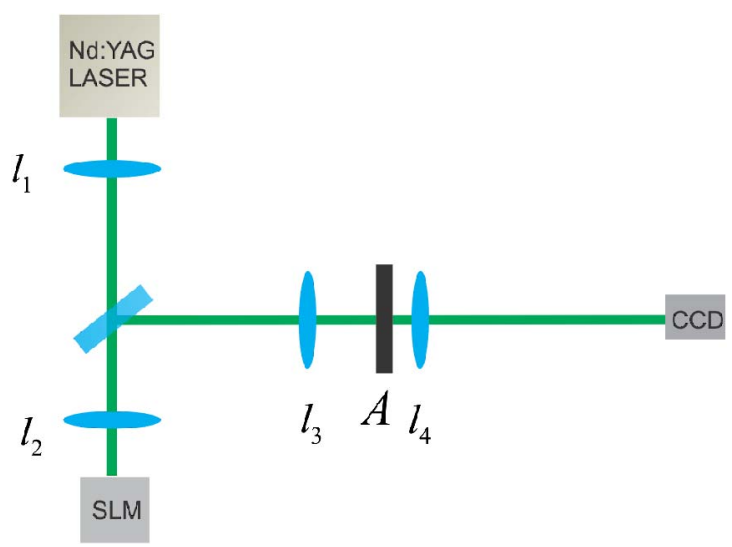

Fig. 1. Experimental setup: $A$ is an aperture (triangular or square), $l_{i}$ are lenses, and SLM is the spatial light modulator. 
The experimental setup is shown schematically in Fig. 1. An Nd:YAG laser operating at $532 \mathrm{~nm}$ illuminates a computer generated hologram [21] with controllable pixels written in a Hamamatsu Model X10468-01 spatial light modulator (SLM) and produces high-order LG modes. A mask with a square or triangular aperture can be superimposed over the LG beam after lens $l_{3}$. The beam at the aperture plane is optically Fourier transformed by lens $l_{4}$ and displayed in a charge coupled device (CCD) camera.

For simplicity, we have used LG beams of light whose transversal profile can be written as

$$
E_{m}(\rho, \phi)=A \rho^{m} \exp (i m \phi),
$$

where $\rho$ and $\phi$ are the polar coordinates, $A$ is a normalization constant, and $m$ is the TC.

We want to determine the Fraunhofer diffraction pattern in the far field region of a beam carrying OAM scattered using a triangular or a square aperture. The diffracted field is given by a Fraunhofer integral [22]

$$
E_{f}\left(\vec{k}_{\perp}\right)=\int_{-\infty}^{+\infty} \tau\left(\vec{r}_{\perp}\right) E_{i}\left(\vec{r}_{\perp}\right) e^{-i \vec{k}_{\perp} \cdot \vec{r}_{\perp}} \mathrm{d} \vec{r} .
$$

This integral gives the far field distribution of electrical field, $E_{f}$, as a Fourier transform of the product of the function describing an aperture, $\tau$, and the incident field, $E_{i}$. Note that the transverse wave vector $\vec{k}_{\perp}$ can be associated with the coordinate of a generic point in the far field plane playing the role of reciprocal space. For a square aperture, this integral can be evaluated analytically.

The field at the aperture plane could be written as $E=A \rho^{m} e^{i m \phi}=A(x \pm i y)^{|m|}$, where we choose the positive sign for positive TC and the negative sign for negative TC. The Fraunhofer diffraction integral for a square aperture becomes

$$
\begin{aligned}
E_{ \pm|m|}\left(k_{x}, k_{y}\right)= & A \int_{-\frac{a}{2}}^{+\frac{a}{2}} \int_{-\frac{a}{2}}^{+\frac{a}{2}}(x \pm i y)^{|m|} \\
& \times \exp \left[-i\left(k_{x} x+k_{y} y\right)\right] \mathrm{d} x \mathrm{~d} y,
\end{aligned}
$$

where $a$ is the length of the square aperture side. This integral can be written as

$$
\begin{aligned}
& E_{m}\left(k_{x}, k_{y}\right) \\
& =A\left(i \frac{\partial}{\partial k_{x}}-\frac{\partial}{\partial k_{y}}\right)^{m} \int_{-\frac{a}{2}}^{+\frac{a}{2}} \int_{-\frac{a}{2}}^{+\frac{a}{2}} \exp \left[-i\left(k_{x} x+k_{y} y\right)\right] \mathrm{d} x \mathrm{~d} y \\
& =A\left(i \frac{\partial}{\partial k_{x}}-\frac{\partial}{\partial k_{y}}\right)^{m} \frac{2 \sin \left(a k_{x} / 2\right)}{k_{x}} \frac{2 \sin \left(a k_{y} / 2\right)}{k_{y}}
\end{aligned}
$$

where we have considered a positive TC. Equation (4) is an analytical result for the diffraction pattern corresponding to any TC. A similar analytical result is not straightforward for a triangular aperture because in this case the integration limits are not all constants. Therefore, we use numerical Fraunhofer diffraction integral in all theoretical results we have presented here.
Figure 2 presents the theoretical results comprising some values of the TC that we have measured ( $m=18,-19$, and 20). Clearly, we cannot define any extern side of the triangular optical lattice with $N$ lobes well defined in order to obtain the TC modulus. However, it still can be used to determine the TC sign. The pattern obtained with negative TC value is rotated by $180^{\circ} \mathrm{com}-$ paring to the positive one. Notwithstanding, the square aperture can be used to determine the TC modulus up to $m=20$. In addition, we have noticed well-defined relations between the TC values and the number of spots in any extern side of the pattern, namely, $m=2 N-2$ for even TC value and $m=2 N-1$ for odd TC value. It is possible to decide if the pattern corresponds to an even or odd value of the TC observing the central region of each pattern. This difference in the pattern formation have been explained previously [20].

In order to have a better understanding about the results presented in Fig. 2, we superimpose the contour plots of the diffraction pattern of each edge (colorful contour) composing the square and triangular apertures, with the interference pattern of whole object (white contour), see Fig. 3. Representations of the square and triangular apertures, of the same side length, are shown in Figs. 3(a) and 3(b), respectively, with each edge being represented by different color so that the colorful contours of Fig. 3(c)-3(f) represent the diffracted pattern by its respective colorful edge. Figures $3(\mathrm{c})-3(\mathrm{~d})$ show the colorful and white contours for $m=0$. It is evident as a bright point on the center of the intensity interference pattern. This point coincides with the intersection of the colorful contour. Figures 3(e) $-3(\mathrm{f})$ show the colorful and white contours for $m=4$. Now, each pattern from each edge is shifted, and this shift is proportional to TC value [9]. As a consequence, various intersection points emerge. These intersection points coincide with the maxima of interference, similarly for $m=0$. In fact, these intersection points define the optical lattice boundary, and they are responsible for truncating the pattern $[9,10]$. Physically, the interference peaks come from the impossibility of distinguishing between path phase and azimuthal phase [23].

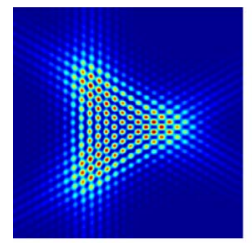

$$
m=18
$$

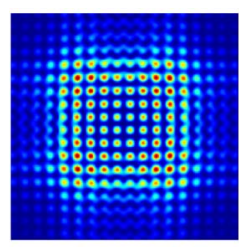

$m=18$

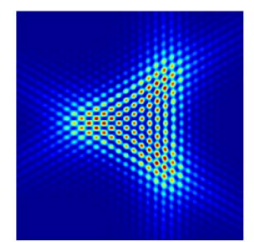

$m=-19$

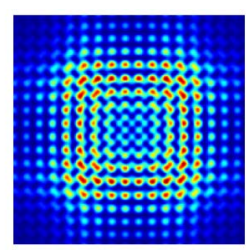

$m=-19$

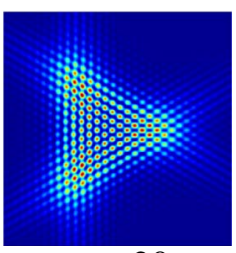

$m=20$

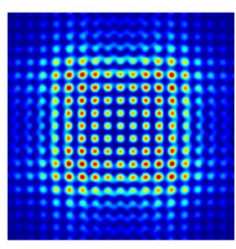

$m=20$
Fig. 2. Diffraction patterns corresponding to the numerical results of Eq. (2) for square and triangular apertures for $m=18,-19$, and 20 . 
(a)

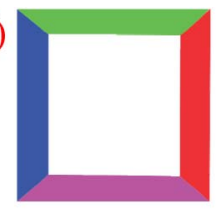

$L$
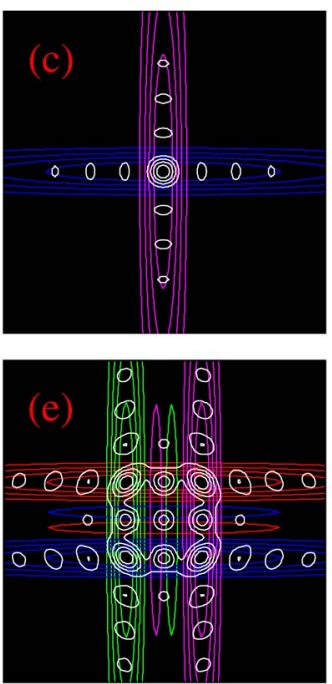

Fig. 3. Diffraction patterns contour plots for a square aperture (left column) and a triangular aperture (right column) shown in white. We also present colorful contour plots for single edges diffraction patterns superimposed in the same figure. The TC for (c) to (d) is $m=0$ and for (e) to (f) is $m=4$.

Figure $\underline{4}$ shows colorful and white contours for $m=20$ for square and triangular apertures. The yellow square shows the optical lattices boundary [Fig. 4(a)], which will be the line of maximum that should define the TC value. It is clear, from Fig. 4 , that the maxima of interference are well formed for the square aperture, which is not the case for the triangular aperture. Observe that the symmetry of square allows one to have more intersection points than the triangular lattice. For the latter case, it is not possible to define a boundary [Fig. 4(b)], which could define the $\mathrm{TC}$ value. It is also interesting to notice that the number of spots in the external side of the triangular diffraction pattern is $N=m+1$ and in the external side of the square diffraction pattern is $N=(m+2) / 2$ or $N=(m+1) / 2$, for even or odd TC values, respectively. For $m \gg 1$, these relations reduce to $N \simeq m$ (triangular aperture) and $N \simeq m / 2$ (square aperture). Similarly, the total number of points in the square lattice of odd TC is, roughly, $N^{2}=$ $(m+1)^{2} / 4 \simeq m^{2} / 4$ and for the triangular lattice is $(m+1)(m+2) / 2 \simeq m^{2} / 2$. This way, for the same side length of apertures, in the reciprocal space, the optical lattice has more points in the triangular pattern than in the square pattern. Therefore, we notice an interesting relation between these two regular polygonal diffractions patterns: We can measure the TC value using a triangular aperture up to $m=10$ and using a square aperture up to $m=20$.

The experimental results are summarized in Fig. 5 for $m=18,19$, and 20 . The experimental results confirm our theoretical results, i.e., we observe that the triangular
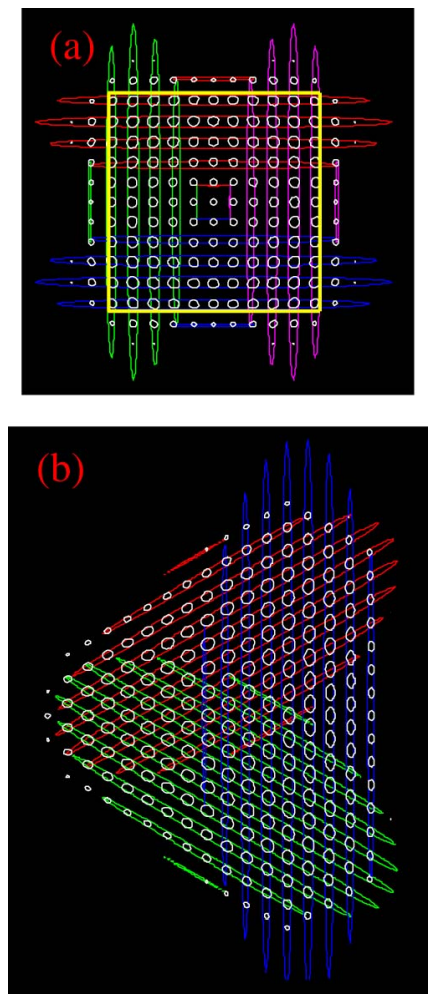

Fig. 4. Diffraction patterns contour plots for (a) square aperture and (b) triangular aperture shown in white. We also present colorful contour plots for single edges diffraction patterns superimposed in the same figure. The TC is $m=20$ for both cases.

aperture does not reveal the TC modulus. However, it still can be used to determine the TC sign. Remarkably, the square aperture can be used to determine the TC modulus, at least, up to $m=20$.

In conclusion, we presented a comparative study of the diffraction problem of light with OAM using two apertures: square and triangle. By observing in the Fraunhofer plane, we showed that with the square aperture it is possible to measure up to 20 the value of TC. This value outperforms two times that obtained with the triangular aperture. Because of symmetry of square, it is possible to build an optical lattice with intersection

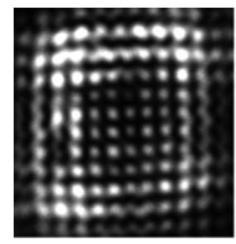

$m=18$

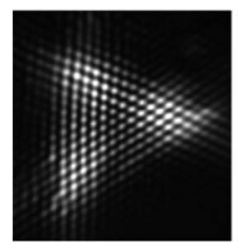

$m=18$

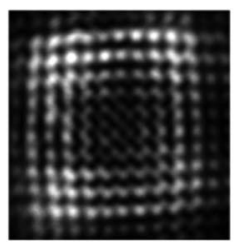

$m=19$

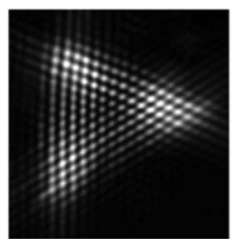

$m=19$

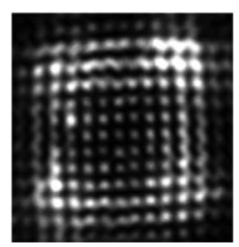

$m=20$

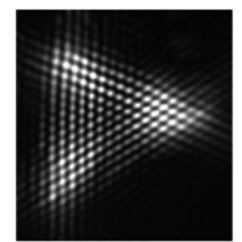

$m=20$
Fig. 5. Experimental results to the Fraunhofer diffraction pattern by a square (top) and triangular apertures (bottom). 
points, resulting in well-defined maxima of interferences. These points define the optical lattice boundary, enabling to determine the amount of OAM. We realized that, even though the optical square lattice is not enough to determine the TC sign, it is possible to measure high order of modulus and sign of TC, up to 20, combining patterns of the triangular and square apertures. It is important to point out that the maxima values of TCs of 20 , for the square aperture, and 10, for the triangular aperture, depend on the experimental arrangement. Specifically, in our case the pattern continuously becomes blurry for higher values than the mentioned above.

The authors are thankful for the financial support from CAPES, CNPq/MCT, Pronex/FAPEAL, INCT-IQ, and INCT-Fotonicom.

\section{References}

1. E. Zauderer, J. Opt. Soc. Am. A 3, 465 (1986).

2. J. Durnin, J. J. Miceli, and J. H. Eberly, Phys. Rev. Lett. 58, 1499 (1987).

3. L. Allen, M. W. Beijersbergen, R. J. C. Spreeuw, and J. P. Woerdman, Phys. Rev. A 45, 8185 (1992).

4. J. Courtial, K. Dholakia, L. Allen, and M. J. Padgett, Phys. Rev. A 56, 4193 (1997).

5. A. Mair, A. Vaziri, G. Weihs, and A. Zeilinger, Nature 412, 313 (2001).

6. D. P. Caetano, M. P. Almeida, P. H. S. Ribeiro, J. A. O. Huguenin, B. C. dos Santos, and A. Z. Khoury, Phys. Rev. A 66, 041801(R) (2002).
7. J. W. R. Tabosa and D. V. Petrov, Phys. Rev. Lett. 83, 4967 (1999).

8. W. J. Condell, J. Opt. Soc. Am. A 2, 206 (1985).

9. Q. S. Ferreira, A. J. Jesus-Silva, E. J. S. Fonseca, and J. M. Hickmann, Opt. Lett. 36, 3106 (2011).

10. J. M. Hickmann, E. J. S. Fonseca, W. C. Soares, and S. Chavez-Cerda, Phys. Rev. Lett. 105, 053904 (2010).

11. D. G. Grier, Nature 424, 810 (2003).

12. G. Molina-Terriza, J. P. Torres, and L. Torner, Nat. Phys. 3, 305 (2007).

13. L. Veissier, A. Nicolas, L. Giner, D. Maxein, A. S. Sheremet, E. Giacobino, and J. Laurat, Opt. Lett. 38, 712 (2013).

14. N. Bozinovic, Y. Yue, Y. X. Ren, M. Tur, P. Kristensen, H. Huang, A. E. Willner, and S. Ramachandran, Science 340, 1545 (2013)

15. V. D'Ambrosio, N. Spagnolo, L. Del Re, S. Slussarenko, Y. Li, L. C. Kwek, L. Marrucci, S. P. Walborn, L. Aolita, and F. Sciarrino, Nat. Commun. 4, 2432 (2013).

16. H. I. Sztul and R. R. Alfano, Opt. Lett. 31, 999 (2006).

17. A. Kumar, P. Vaity, and R. P. Singh, Opt. Commun. 283, 4141 (2010).

18. D. P. Ghai, P. Senthilkumaran, and R. S. Sirohi, Opt. Laser Eng. 47, 123 (2009).

19. G. C. G. Berkhout and M. W. Beijersbergen, Phys. Rev. Lett. 101, 100801 (2008).

20. P. H. F. Mesquita, A. J. Jesus-Silva, E. J. S. Fonseca, and J. M. Hickmann, Opt. Express 19, 20616 (2011).

21. J. P. Kirk and A. L. Jones, J. Opt. Soc. Am. 61, 1023 (1971)

22. J. W. Goodman, Introduction to Fourier Optics, 2nd ed. (McGraw-Hill, 1996).

23. J. M. Hickmann, E. J. S. Fonseca, and A. J. Jesus-Silva, Europhys. Lett. 96, 64006 (2011). 